Pak. j. sci. ind. res. Ser. A: phys. sci. 201356 (3) 131-137

\title{
Microwave Assisted Knoevenagel Condensation: Synthesis and Antimicrobial Activities of Some $\alpha$-Cyanoacrylates
}

\author{
Md. Mosharef Hossain Bhuiyan*, Khandker Md. Mizanur Rahman, \\ Md. Ashraful Alam and Md. Mayez Mahmud \\ Department of Chemistry, University of Chittagong, Chittagong 4331, Bangladesh
}

(received June 6, 2012; revised August 14, 2012; accepted November 13, 2012)

\begin{abstract}
A series of $\alpha$-cyanoacrylates (1-9) were prepared by Knoevenagel condensation of ethyl cyanoacetate with corresponding aromatic aldehydes in presence of ammonium acetate $\left(\mathrm{NH}_{4} \mathrm{OAc}\right)$ using microwave irradiation under solvent free condition. The reactions were completed in 20-60 sec with excellent yield. These compounds were screened for their antibacterial activities against five pathogenic organisms: Bacillus cereus, Staphylococcus aureus (ATCC 6538), Vibrio cholerae, Shigella dysenteriae (AE 14396) and Salmonella typhi (AE 14612) and antifungal activity against two organisms: Aspergillus flavus and Saccharomyces cerevisiae using disc diffusion method and poisoned-food technique, respectively. Some of them were found to possess significant activity, when compared to standard drugs.
\end{abstract}

Keywords: Knoevenagel condensation, microwave irradiation, $\alpha$-cyanoacrylates, antimicrobial activity

\section{Introduction}

Carbon-carbon double bond formation (Shen, 1998) is one of the useful and fundamental reactions in synthetic organic chemistry, particularly in the synthesis of complex natural products with biological activity. The Knoevenagel condensation is one of the most important, useful and widely employed methods for the formation of carbon-carbon bonds. It has been used for the preparation of a range of substituted alkenes and bioactive molecules, as a key step in natural product synthesis and important intermediate for the synthesis of cosmetic, perfume, agrochemicals and pharmaceuticals (Tietze and Beifuss, 1991). Active methylene compounds such as malononitrile, ethyl cyanoacetate, malonic ester etc., are largely used in the Knoevenagel condensation. The ylidenemalononitriles thus obtained have found increasing applications in industry, agriculture, medicine, biological science and in the elegant synthesis of fine chemicals (Freeman, 1980; Fatiadi, 1978a, 1978b). They are important intermediates for the synthesis of various organic compounds, mainly by cyclization reactions (Campaigne and Schneller, 1976). Knoevenagel condensation products with ethyl cyanoacetate have been utilized successfully in the synthesis of mercaptopyrimidine as an intermediate for an antimetabolite, substrates for Guareschi reaction, indanone derivatives for diterpene alkaloid, cyano-

*Author for correspondence; E-mail: mosharef65@yahoo.com coumarins (Kumar, 2009; Bigi et al., 1999) and versatile precursors for the synthesis of substituted heterocycles (Kandeel et al., 1996).

The Knoevenagel condensation is generally carried out in the presence of weak organic bases such as aliphatic amines, ethylenediamine and piperidine or their corresponding ammonium salts, Lewis bases and acids including $\mathrm{ZnCl}_{2}, \mathrm{CdI}_{2}, \mathrm{TiCl}_{4}, \mathrm{Al}_{2} \mathrm{O}_{3}, \mathrm{MgO}, \mathrm{ZnO}$, $\mathrm{AlPO}_{4}-\mathrm{Al}_{2} \mathrm{O}_{3}, \mathrm{KF}-\mathrm{Al}_{2} \mathrm{O}_{3}$, natural hydroxyapatite $\left(\mathrm{Ca}_{10}\left(\mathrm{PO}_{4}\right)_{6}(\mathrm{OH})_{2}\right)$ (Mallouk et al., 2010), tetra- $n$ butylammonium hydroxide (TBAH) (Balalaie and Bararjanian, 2006), $N$-methylpiperazine (Mukopadhyay and Datta, 2008), 1,1,3,3-tetramethyl-guanidium lactate (Zhang et al., 2006), polyacrylonitrile fibre (Li et al., 2011), Imidazolium chloride immobilized SBA-15 (Parvin et al., 2012) and ammonium acetate $\left(\mathrm{NH}_{4} \mathrm{OAc}\right)$ basic alumina (Balalaie and Nemati, 2000). However, this employs hazardous benzene and requires prolonged heating with continuous water removal. The use of such bases/acids and solvents has led to environmental problems, i.e., the necessity to dispose of huge amounts of organic waste due to the formation of undesirable side products. Ionic liquid, recyclable catalysts (Ye et al., 2011), electrochemical (Feroci et al., 2007), microwave (Saha et al., 2008) and ultrasound (Li et al., 2004) activation methods have also been reported.

Recently microwave radiation, a non-conventional energy source, for the activation of reactions has gained 
the attention of chemists due to its unique advantages, such as shorter reaction times, cleaner reaction products, higher yields and better selectivities (Kappe, 2004; Lidstrom et al., 2001; Varma, 1999). Moreover, the combination of MW activation and solvent free conditions leads to enhanced conversion rates, higher yields, easier workup and in general cleaner reactions, therefore, confirming the real advantages of this approach in the framework of green chemistry.

In continuation to earlier studies directed at the development of practical and efficient chemical processes (Bhuiyan et al., 2012; 2011; Basu et al., 2003), a sustainable Knoevenagel condensation protocol was reported using microwave irradiation (MWI) and ammonium acetate $\left(\mathrm{NH}_{4} \mathrm{OAc}\right)$ catalysis under solventfree conditions for the preparation of $\alpha$-cyanoacrylates. The obtained compounds were screened for their antimicrobial activity.

\section{Materials and Methods}

Melting point was recorded with electro thermal melting point apparatus. Thin layer chromatography was performed on Kieselgel $\mathrm{GF}_{254}$ and visualisation was accomplished by iodine vapour or uv flame. The infrared (IR) spectra were recorded by FTIR spectrophotometer (Model-8900, Shimadzu, Japan) using $\mathrm{KBr}$ matrix in the range 4000-200 $\mathrm{cm}^{-1} .{ }^{1} \mathrm{H}-\mathrm{NMR}$ (400 MHz and $500 \mathrm{MHz}$ ) and ${ }^{13} \mathrm{C}$-NMR (100 MHz and $125 \mathrm{MHz}$ ) spectra were recorded on JEOL GS $\times 400$, GEOL JNM-AL 400 (400 $\mathrm{MHz})$ and JEOL GS $\times 400$, GEOL JNM-AL $400(100$ $\mathrm{MHz}$ ) spectrometer in $\mathrm{CDCl}_{3}, \mathrm{CD}_{3} \mathrm{OD}$ and DMSO-d $\mathrm{d}_{6}$ as solvent. Chemical shifts were reported in $\delta$ unit (ppm) with reference to TMS as an internal standard and $J$ values are given in $\mathrm{Hz}$. The carbon, hydrogen and nitrogen percentages in synthesised products were analysed according to the approved method ASTM D-5291 by employing Leco-CHNS-932 analyzer. All reactions were carried out in a commercially available LG microwave oven (MB - 3947C) having a maximum power output of $800 \mathrm{~W}$ operating at $2450 \mathrm{MHz}$.

Syntheses. General procedure for the synthesis of $\alpha$-cyanoacrylates. To an equimolar mixture of aromatic aldehyde and ethyl cyanoacetate, catalytic amount of ammonium acetate was added and the reaction mixture irradiated under microwave condition at 160-320 watt for 20-60 sec. After complete conversion of the reaction (TLC; ethyl acetate: $n$-hexane; $1: 5, \mathrm{v} / \mathrm{v}$ ), the obtained solid mass was recrystallised from ethyl acetate and $n$-hexane solvent mixture.
Spectral data. Ethyl 2-cyano-3-(2-methylphenyl)acrylate (1). Off-white crystals, IR $(\mathrm{KBr}) \mathrm{v}_{\max }\left(\mathrm{cm}^{-1}\right)$ : 3182-2981 (C-H), $2219.91(\mathrm{~s}, \mathrm{C} \equiv \mathrm{N}), 1712.67(\mathrm{~s}, \mathrm{C}=\mathrm{O}$, ester), 1591.16 ( $\mathrm{s}, \mathrm{C}=\mathrm{C}$ ), 1112.85 (s, C-O str, ether). ${ }^{1} \mathrm{H}-\mathrm{NMR}\left(400 \mathrm{MHz}, \mathrm{CD}_{3} \mathrm{OD}\right): \delta 8.62(\mathrm{~s}, 1 \mathrm{H},=\mathrm{CH})$, 8.05 (d, 1H, H-6', $J=7.80 \mathrm{~Hz}), 7.45$ (m, 1H, H-3'), 7.33 (m, 2H, H-4', H-5'), 4.37 (q, 2H, $\mathrm{OCH}_{2}, J=7.32$ $\mathrm{Hz}), 2.44\left(\mathrm{~s}, 3 \mathrm{H}, \mathrm{CH}_{3}\right), 1.38\left(\mathrm{t}, 3 \mathrm{H}, \mathrm{OCH}_{2} \mathrm{CH}_{3}, J=7.32\right.$ $\mathrm{Hz}) .{ }^{13} \mathrm{C}-\mathrm{NMR}\left(100.40 \mathrm{MHz}, \mathrm{CD}_{3} \mathrm{OD}\right): \delta 163.50$, $154.47,141.04,133.80,132.08,131.99,129.39,127.52$, $116.17,105.89,63.71,19.73,14.42$. DEPT - $90(100.40$ $\left.\mathrm{MHz}, \mathrm{CD}_{3} \mathrm{OD}\right): \delta 154.47,133.81,132.08,129.40$, 127.52. DEPT - 135 (100.40 MHz, $\left.\mathrm{CD}_{3} \mathrm{OD}\right): \delta 154.47$, $133.81,132.08,129.40,127.52,63.72\left(\mathrm{CH}_{2}\right)$, 19.73 $\left(\mathrm{CH}_{3}\right), 14.42\left(\mathrm{CH}_{3}\right)$. Analysis calculated for $\mathrm{C}_{13} \mathrm{H}_{13} \mathrm{NO}_{2}$ (215.25): $\mathrm{C}, 72.54 ; \mathrm{H}, 6.09 ; \mathrm{N}, 6.51$; found: C, 73.25; H, 5.68; N, 6.15 .

Ethyl 2-cyano-3-(3-methyphenyl)-acrylate (2). Offwhite crystals, IR (KBr) $\cup_{\max }\left(\mathrm{cm}^{-1}\right): 3184,2925$ (CH), 2217.99 (s, $\mathrm{C} \equiv \mathrm{N}), 1726.17$ (, $\mathrm{C}=\mathrm{O}$, ester), 1606.59 (s, C=C), 1583.59 (s, C=C, Ph), 1178.43(s, C-O str.). $1 \mathrm{H}-\mathrm{NMR}\left(400 \mathrm{MHz}, \mathrm{CDCl}_{3}\right): \delta 8.23(\mathrm{~s}, 1 \mathrm{H},=\mathrm{CH})$, 7.82 (d, 1H, H-6' $J=7.32 \mathrm{~Hz}), 7.78$ (s, 1H, H-2'), 7.41 (m, 2H, H-4', H-5'), 4.39 (q, 2H, $\mathrm{OCH}_{2}, J=7.32 \mathrm{~Hz}$ ), $2.42\left(\mathrm{~s}, 3 \mathrm{H}, \mathrm{CH}_{3}\right), 1.41\left(\mathrm{t}, 3 \mathrm{H}, \mathrm{OCH}_{2} \mathrm{CH}_{3}, J=7.32\right.$ $\mathrm{Hz}) .{ }^{13} \mathrm{C}-\mathrm{NMR}\left(100.40 \mathrm{MHz}, \mathrm{CDCl}_{3}\right): \delta 162.53$, 155.21, 139.06, 134.17, 131.63, 131.38, 129.12, 128.17, $115.51,102.55,62.63,21.23,14.11$. DEPT - 135 $\left(100.40 \mathrm{MHz}, \mathrm{CDCl}_{3}\right): \delta 155.21,134.17,131.64$, 129.11, 128.17, $62.64\left(\mathrm{CH}_{2}\right), 21.25\left(\mathrm{CH}_{3}\right), 14.12$ $\left(\mathrm{CH}_{3}\right)$. Analysis calculated for $\mathrm{C}_{13} \mathrm{H}_{13} \mathrm{NO}_{2}(215.25)$ : C, 72.54; H, 6.09; N, 6.51; found: C, 73.14; H, 5.69; $\mathrm{N}, 5.39$.

Ethyl 2-cyano-3-(4-hydroxyphenyl)-acrylate (3). Light yellow crystals, IR $(\mathrm{KBr}) \cup_{\max }\left(\mathrm{cm}^{-1}\right): 3257.55(\mathrm{~b}, \mathrm{OH})$, 3161-2900 (C-H), $2231.49(\mathrm{~s}, \mathrm{C} \equiv \mathrm{N}), 1731.96$ (s, C=O, ester), $1587.31(\mathrm{~s}, \mathrm{C}=\mathrm{C}), 1519.81(\mathrm{~s}, \mathrm{C}=\mathrm{C}, \mathrm{Ph}), 1089.71$ (s, C-O str, ether). ${ }^{1} \mathrm{H}-\mathrm{NMR}\left(400 \mathrm{MHz}, \mathrm{CD}_{3} \mathrm{OD}\right): \delta$ $8.20(\mathrm{~s}, 1 \mathrm{H},=\mathrm{CH}), 7.96\left(\mathrm{~d}, 2 \mathrm{H}, \mathrm{H}-2^{\prime}, \mathrm{H}-6{ }^{\prime}, J=9.16\right.$ Hz), 6.90 (d, 2H, H-3', H-5', J=8.72 Hz), 4.33 (q, 2H, $\mathrm{OCH}_{2}, J=7.32 \mathrm{~Hz}$ ), 1.36 (t, $3 \mathrm{H}, \mathrm{OCH}_{2} \mathrm{CH}_{3}, J=7.32$ $\mathrm{Hz}) .{ }^{13} \mathrm{C}-\mathrm{NMR}\left(100.40 \mathrm{MHz}, \mathrm{CD}_{3} \mathrm{OD}\right): \delta 164.51$, $164.37,155.89,135.12,133.64,124.43,117.32,117.26$, 116.84, 98.84, 63.30, 14.47. DEPT - $90(100.40 \mathrm{MHz}$, $\left.\mathrm{CD}_{3} \mathrm{OD}\right): \delta 155.90,135.12,117.26$. DEPT - $135(100.40$ $\left.\mathrm{MHz}, \mathrm{CD}_{3} \mathrm{OD}\right): \delta 155.90,136.12,117.26,63.30\left(\mathrm{CH}_{2}\right)$, $14.47\left(\mathrm{CH}_{3}\right)$. Analysis calculated for $\mathrm{C}_{12} \mathrm{H}_{11} \mathrm{NO}_{3}$ (217.23): C, 66.35; H, 5.10; N, 6.45; found: $\mathrm{C}, 67.11$; $\mathrm{H}, 4.94 ; \mathrm{N}, 6.45$. 
Ethyl 2-cyano-3-(4-hydroxy-3-methoxyphenyl)-acrylate (4). Yellow crystals, IR (KBr) $v_{\max }\left(\mathrm{cm}^{-1}\right): 3375.20$ (b, $\mathrm{OH}), 3128.32$, 2931.60 (C-H), $2217.99(\mathrm{~s}, \mathrm{C} \equiv \mathrm{N}), 1704.96$ (s, $\mathrm{C}=\mathrm{O}$, ester), 1573.81 (s, $\mathrm{C}=\mathrm{C}), 1508.23$ (s, $\mathrm{C}=\mathrm{C}, \mathrm{Ph}$ ), 1172.64 (s, C-O str, ether). ${ }^{1} \mathrm{H}-\mathrm{NMR}(500 \mathrm{MHz}$, DMSO$\left.\mathrm{d}_{6}\right): \delta 8.20(\mathrm{~s}, 1 \mathrm{H},=\mathrm{CH}), 7.74\left(\mathrm{~s},{ }^{1} \mathrm{H}, \mathrm{H}-2^{\prime}\right), 7.60(\mathrm{~d}, 1 \mathrm{H}$, H-6', $J=8.55 \mathrm{~Hz}$ ), 6.94 (d, 1H, H-5', $J=8.00 \mathrm{~Hz}), 4.27$ (q, $\left.2 \mathrm{H},-\mathrm{OCH}_{2}, J=6.90 \mathrm{~Hz}\right), 3.81\left(\mathrm{~s}, 3 \mathrm{H}, \mathrm{OCH}_{3}\right), 1.28$ (t, $\left.3 \mathrm{H}, \mathrm{OCH}_{2} \mathrm{CH}_{3}, J=6.90 \mathrm{~Hz}\right) .{ }^{13} \mathrm{C}-\mathrm{NMR}(100.40 \mathrm{MHz}$, $\mathrm{CDCl}_{3}$ ): $\delta 162.65,154.93,152.78,147.82,127.20,122.82$, $116.65,116.02,114.05,96.95,61.94,55.56,14.07$. DEPT - 135 (125 MHz, DMSO-d 6 ): $\delta 154.93,127.20,116.03$, 114.04, $61.95\left(\mathrm{CH}_{2}\right), 55.576\left(\mathrm{OCH}_{3}\right), 14.07\left(\mathrm{CH}_{3}\right)$. Analysis calculated for $\mathrm{C}_{13} \mathrm{H}_{13} \mathrm{NO}_{4}$ (247.25): C, 63.15; H, 5.30; N, 5.66; found: C, 62.50; H, 3.75; N 5.29.

Ethyl 2-cyano-3-(2,4-dimethoxyphenyl)-acrylate (5). Orange crystals, IR (KBr) $v_{\max }\left(\mathrm{cm}^{-1}\right): 3110-2840$ (CH), $2214.13(\mathrm{~s}, \mathrm{C} \equiv \mathrm{N}), 1714.60$ ( $\mathrm{s}, \mathrm{C}=\mathrm{O}$, ester), 1610.45 (s, C=C), 1581.52 (s, C=C, Ph), 1126.35 (s, C-O str, ether). ${ }^{1} \mathrm{H}-\mathrm{NMR}\left(500 \mathrm{MHz}, \mathrm{CDCl}_{3}\right): \delta 8.70(\mathrm{~s}, 1 \mathrm{H},=\mathrm{CH})$, 8.40 (d, 1H, H-6', $J=8.60$ Hz), 6.60 (d, 1H, H-5', $J=$ $9.15 \mathrm{~Hz}$ ), 6.44 (s, 1H, H-3'), 4.37 (q, $2 \mathrm{H}, \mathrm{OCH}_{2}, J=7.26$ $\mathrm{Hz}), 3.89\left(\mathrm{~s}, 6 \mathrm{H}, 2 \times \mathrm{OCH}_{3}\right), 1.38\left(\mathrm{t}, 3 \mathrm{H}, \mathrm{OCH}_{2} \mathrm{CH}_{3}, J=\right.$ $6.90 \mathrm{~Hz}) .{ }^{13} \mathrm{C}-\mathrm{NMR}\left(125.00 \mathrm{MHz}, \mathrm{CDCl}_{3}\right): \delta 165.63$, $163.47,161.32,148.59,131.00,116.82,113.92,105.96$, 98.01, 97.93, 62.10, 55.72, 55.63, 14.16. DEPT - 90 (125 $\left.\mathrm{MHz}, \mathrm{CDCl}_{3}\right): \delta 148.58,131.00,105.96,97.94$. DEPT - 135 (100.40 MHz, $\left.\mathrm{CDCl}_{3}\right): \delta 148.59,131.00,105.96$, 97.94, $62.10\left(\mathrm{CH}_{2}\right), 55.72\left(\mathrm{OCH}_{3}\right), 55.64\left(\mathrm{OCH}_{3}\right), 14.16$ $\left(\mathrm{CH}_{3}\right)$. Analysis calculated for $\mathrm{C}_{14} \mathrm{H}_{15} \mathrm{NO}_{4}$ (261.28): $\mathrm{C}$, 64.36; H, 5.79; N, 5.36; found: C, 64.72; H, 5.85; N, 6.03 .

Ethyl 2-cyano-3-(3-nitrophenyl)-acrylate (6). Off-white crystals, IR (KBr) $v_{\max }\left(\mathrm{cm}^{-1}\right): 3114-2870(\mathrm{C}-\mathrm{H}), 2225.70$ (s, C $\equiv \mathrm{N}), 1718.46$ (s, C=O, ester), 1606.59 (s, C=C), 1571.88 (s, $\mathrm{C}=\mathrm{C}, \mathrm{Ph}), 1529.45$ ( $\mathrm{NO}_{2}$, sym. str.), 1473.51 ( $\mathrm{NO}_{2}$, asym. str.), 1207.36 (s, C-O str.). ${ }^{1} \mathrm{H}-\mathrm{NMR}$ (400 $\left.\mathrm{MHz}, \mathrm{CD}_{3} \mathrm{OD}\right): \delta 8.91(\mathrm{~s}, 1 \mathrm{H},=\mathrm{CH}), 8.47(\mathrm{~s}, 1 \mathrm{H}, \mathrm{H}-$ 2'), 8.44 (d, 1H, H-4', J=8.24 Hz), 8.38 (d, 1H, H-6', $J=8.24 \mathrm{~Hz}$ ), 7.81 (t, 1H, H-5', $J=8.24 \mathrm{~Hz}), 4.40$ (q, $\left.2 \mathrm{H}, \mathrm{OCH}_{2}, J=7.32 \mathrm{~Hz}\right), 1.39\left(\mathrm{t}, 3 \mathrm{H}, \mathrm{OCH}_{2} \mathrm{CH}_{3}, J=\right.$ $7.32 \mathrm{~Hz}) .{ }^{13} \mathrm{C}-\mathrm{NMR}\left(100.40 \mathrm{MHz}, \mathrm{DMSO}_{6}\right): \delta 163.18$, 158.41, 146.87, 143.24, 141.29, 140.92, 137.51, 135.34, 125.46, 115.91, 73.06, 16.06. Analysis calculated for $\mathrm{C}_{12} \mathrm{H}_{10} \mathrm{~N}_{2} \mathrm{O}_{4}$ (246.22): C, 58.54; H, 4.09; N, 25.99; found: C, 58.71; H, 3.58; N, 12.03.

Ethyl 2-cyano-3-(9-anthracenyl)-acrylate (7). Yellow crystals, IR (KBr) $v_{\max }\left(\mathrm{cm}^{-1}\right): 2981.74(\mathrm{C}-\mathrm{H}), 2227.63$ (s, C $\equiv \mathrm{N}), 1724.24$ (s, C=O, ester), 1612.38 (s, C=C), 1060.78 (C-O). ${ }^{1} \mathrm{H}-\mathrm{NMR}\left(500 \mathrm{MHz}, \mathrm{CDCl}_{3}\right): \delta 9.32$ (s, 1H, H-10'), 8.60 (s, 1H, =CH), 8.08 (d, 2H, H-1', H-8', $J=8.55 \mathrm{~Hz}$ ), 7.97 (d, 2H, H-4', H-5', $J=8.60$ $\mathrm{Hz}$ ), 7.60 (d, 2H, H-2', H-7', $J=7.40 \mathrm{~Hz}$ ), 7.56 (d, $2 \mathrm{H}, \mathrm{H}-3$ ', H-6', $J=8.05 \mathrm{~Hz}$ ), 4.51 (q, 2H, $\mathrm{OCH}_{2}, J=$ $6.85 \mathrm{~Hz}), 1.49$ (t, $\left.3 \mathrm{H}, \mathrm{OCH}_{2} \mathrm{CH}_{3}, J=6.90 \mathrm{~Hz}\right) .{ }^{13} \mathrm{C}-$ NMR $\left(125 \mathrm{MHz}, \mathrm{CDCl}_{3}\right): \delta 161.53,155.42,130.95$, 130.83, 129.21, 128.95, 127.35, 125.71, 124.99, 124.54, 114.09, 112.77, 63.01, 14.17. DEPT - 90 (125 MHz, $\left.\mathrm{CDCl}_{3}\right): \delta 155.43,130.83,129.21,127.35,125.72$, 124.54. DEPT - $135\left(125 \mathrm{MHz}, \mathrm{CDCl}_{3}\right): \delta 155.43$, $130.83,129.21,127.35,125.72,124.54,62.02\left(\mathrm{CH}_{2}\right)$, $14.18\left(\mathrm{CH}_{3}\right)$. Analysis calculated for $\mathrm{C}_{20} \mathrm{H}_{15} \mathrm{NO}_{2}$ (301.35): C, 79.72; H, 5.02; N, 4.65; found: C, 79.38; H, 5.31; N, 5.16 .

Ethyl 2-cyano-3-(4-N,N-dimethylaminophenyl)-acrylate (8). Yellow crystals, IR (KBr) $\cup_{\max }\left(\mathrm{cm}^{-1}\right): 3089.75$ (=CH), 2985.60 (C-H), 2208.34 (s, C $\equiv N), 1704.96$ (s, C=O, ester), $1612.38(\mathrm{~s}, \mathrm{C}=\mathrm{C}), 1569.95(\mathrm{~s}, \mathrm{C}=\mathrm{C}, \mathrm{Ph}), 1168.78$ (s, C-O str, ether). ${ }^{1} \mathrm{H}-\mathrm{NMR}\left(400 \mathrm{MHz}, \mathrm{CDCl}_{3}\right.$ ): $\delta 8.08$ $(\mathrm{s}, 1 \mathrm{H},=\mathrm{CH}), 7.94$ (d, 2H, H-2', H-6', $J=9.16 \mathrm{~Hz}), 6.70$ (d, 2H, H-3', H-5', J=9.60 Hz), 4.34 (q, 2H, $\mathrm{OCH}_{2}$, $J=7.32 \mathrm{~Hz}), 3.11\left(\mathrm{~s}, 6 \mathrm{H}, 2 \times \mathrm{CH}_{3}\right), 1.37\left(\mathrm{t}, 3 \mathrm{H}, \mathrm{OCH}_{2} \mathrm{CH}_{3}\right.$, $J=7.32 \mathrm{~Hz}) .{ }^{13} \mathrm{C}-\mathrm{NMR}\left(100.40 \mathrm{MHz}, \mathrm{CDCl}_{3}\right): \delta 164.27$, 154.52, 153.48, 134.01, 119.28, 117.57, 111.41, 93.88, $61.84,39.98,14.24$. Analysis calculated for $\mathrm{C}_{14} \mathrm{H}_{16} \mathrm{~N}_{2} \mathrm{O}_{2}$ (244.30): C, 68.83; H, 6.60; N, 11.47 ; found : C, 69.43; H, 6.34; N, 11.27.

Ethyl 2-cyano-5-(4-N, N-dimethylaminophenyl)-penta2,4-dienoate (9). Deep pink crystals, IR (KBr) $\mathrm{v}_{\max }$ $\left(\mathrm{cm}^{-1}\right)$ : 3090-2905 (C-H), $2216.06(\mathrm{~s}, \mathrm{C} \equiv \mathrm{N}), 1706.88$ (s, C=O, ester), 1587.31 (s, C=C), 1548.73 (s, C=C, $\mathrm{Ph}), 1153.36$ (C-O). ${ }^{1} \mathrm{H}-\mathrm{NMR}\left(400 \mathrm{MHz}, \mathrm{CDCl}_{3}\right): \delta$ 7.97 (d, 1H, H-3, J=11.88 Hz), 7.49 (d, 2H, H-2', H$\left.6^{\prime}, J=9.12 \mathrm{~Hz}\right), 7.18$ (d, 1H, H-5, $\left.J=12.00 \mathrm{~Hz}\right), 7.11$ (dd, 1H, H-4, J=11.88 Hz, 2.85Hz), 6.67 (d, 2H, H3', H-5', J = 9.16 Hz), 4.31 (q, 2H, $J=7.36 \mathrm{~Hz}, \mathrm{OCH}_{2}$ ), $3.06\left(\mathrm{~s}, 6 \mathrm{H}, 2 \times \mathrm{CH}_{3}\right), 1.36(\mathrm{t}, 3 \mathrm{H}, J=7.32 \mathrm{~Hz}$, $\left.\mathrm{OCH}_{2} \mathrm{CH}_{3}\right) \cdot{ }^{13} \mathrm{C}-\mathrm{NMR}\left(100.40 \mathrm{MHz}, \mathrm{CDCl}_{3}\right): \delta 163.19$, 156.48, 152.23, 150.13, 130.68, 122.37, 117.94, 115.59, $111.65,98.97,61.62,39.93,14.08$. DEPT - 90 (100.40 $\left.\mathrm{MHz}, \mathrm{CD}_{13}\right): \delta 156.48,150.14,130.68,117.94,111.66$. DEPT - 135 (100.40 MHz, $\left.\mathrm{CDCl}_{3}\right): \delta 156.48,150.13$, 130.68, 117.94, 111.66, $61.63\left(\mathrm{CH}_{2}\right), 39.93\left(\mathrm{CH}_{3}\right)$, $39.91\left(\mathrm{CH}_{3}\right), 14.09\left(\mathrm{CH}_{3}\right)$. Analysis calculated for $\mathrm{C}_{16} \mathrm{H}_{18} \mathrm{~N}_{2} \mathrm{O}_{2}$ (270.33): C, 71.09; H, 6.71; N, 10.36; found: C, 71.46; H, 6.39; N, 11.25. 
Table 1. Antibacterial activity of the synthesised compounds (1-9)

\begin{tabular}{llllll}
\hline \hline \multirow{2}{*}{ Compounds } & \multicolumn{5}{c}{ Zone of inhibition in $\mathrm{mm}(100 \mathrm{mg}(\mathrm{dw}) / \mathrm{disc})$} \\
\cline { 2 - 6 } & B. cereus & S. aureus & V. cholerae & S. dysenteriae & S. typhi \\
\hline $\mathbf{1}$ & 7 & -- & 6.5 & -- & 7 \\
$\mathbf{2}$ & -- & -- & 6 & -- & 7.5 \\
$\mathbf{3}$ & 7 & 6 & 6 & 6 & 8 \\
$\mathbf{4}$ & 10 & 6 & 7 & 6.5 & 7 \\
$\mathbf{5}$ & -- & -- & -- & 6.5 & -- \\
$\mathbf{6}$ & 7 & -- & 6 & -- & 8 \\
$\mathbf{7}$ & -- & 7 & -- & - \\
$\mathbf{8}$ & -- & -- & -- & -- & 7.5 \\
$\mathbf{9}$ & 6.5 & 6.5 & 17 & -- & 7.5 \\
Ampicillin & 20 & 12 & & 30 & 24 \\
\hline \hline
\end{tabular}

-- = no inhibition; $\mathrm{dw}=$ dry weight

Antimicrobial screening. The synthesized compounds (1-9) were screened for antibacterial activity against five pathogenic organisms: B. cereus (BTCC 19), S. aureus (ATCC 6538), V. cholerae, S. dysenteriae (AE 14396) and S. typhi (AE 14612 (Table 1) and antifungal activity against two organisms: A. flavus and $S$. cerevisiae (Table 2). The disc diffusion method (Bauer et al., 1966) and poisoned-food technique (Grover and Moore, 1962) were used for antibacterial and antifungal activities, respectively.

The tested compounds were dissolved in N, Ndimethyl formamide (DMF) to get a solution of $1 \mathrm{mg} / \mathrm{mL}$. The inhibition zones were measured in

Table 2. Antifungal activity of the synthesised compounds (1-9).

\begin{tabular}{lll}
\hline \hline Compounds & \multicolumn{2}{l}{$\begin{array}{l}\text { \% Inhibition of mycelial growth } \\
(100 \mu \mathrm{g}(\mathrm{dw}) / \mathrm{mL} \text { PDA })\end{array}$} \\
\cline { 2 - 3 } & A. flavus & S. cerevisiae \\
\hline $\mathbf{1}$ & 62 & $75^{*}$ \\
$\mathbf{2}$ & 71 & $80^{*}$ \\
$\mathbf{3}$ & 64 & $82^{*}$ \\
$\mathbf{4}$ & 71 & $87^{*}$ \\
$\mathbf{5}$ & 71 & $82^{*}$ \\
$\mathbf{6}$ & 62 & $85^{*}$ \\
$\mathbf{7}$ & $75^{*}$ & $90^{*}$ \\
$\mathbf{8}$ & $78^{*}$ & $85^{*}$ \\
$\mathbf{9}$ & $82^{*}$ & $87^{*}$ \\
Nystatin & 90 & 80 \\
\hline \hline
\end{tabular}

* = good inhibition; $\mathrm{dw}=$ dry weight millimeters at the end of an incubation period of $48 \mathrm{~h}$ at $(35 \pm 2)^{\circ} \mathrm{C}$. DMF alone showed no inhibition. Nutrient agar (NA) and potato dextrose agar (PDA) were used as basal media to test the bacteria and fungi, respectively. Commercial antibacterial Ampicillin and antifungal Nystatin were also tested under similar conditions for comparison.

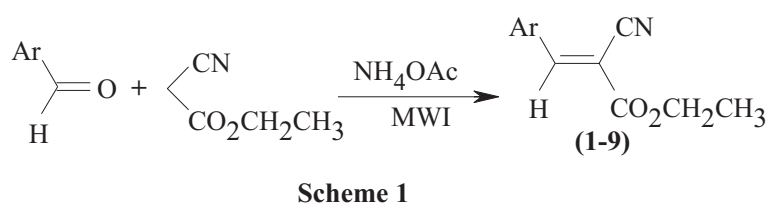

\section{Results and Discussion}

Various methods for the synthesis of $\alpha$-cyanoacrylates have been developed and employed successfully in the light of green chemistry aspects. In the present work, nine $\alpha$-cyanoacrylates derivatives were synthesised from several aromatic aldehydes and active methylene compound ethyl cyanoacetate in the presence of catalytic amount of $\mathrm{NH}_{4} \mathrm{OAc}$ by modified Knoevenagel reaction using microwave irradiation, as indicated in Scheme 1 and Table 3. The corresponding reactions proceeded smoothly and in excellent yields (85-99\%).

An important feature of this procedure is the survival of variety of functional groups such as nitro, chloro, amino and ether under the reaction conditions. The structures of the products were established from their IR, ${ }^{1} \mathrm{H}$ NMR, ${ }^{13} \mathrm{C}$ NMR and elemental analyses. For example the IR spectrum of compound 9 displayed characteristic band absorption for $\mathrm{C}=\mathrm{O}$ of ester functionality at $1706.88 \mathrm{~cm}^{-1}$. The absorption band at 
Table 3. Microwave assisted Knoevenagel reaction between various aldehydes and ethyl cyanoacetate

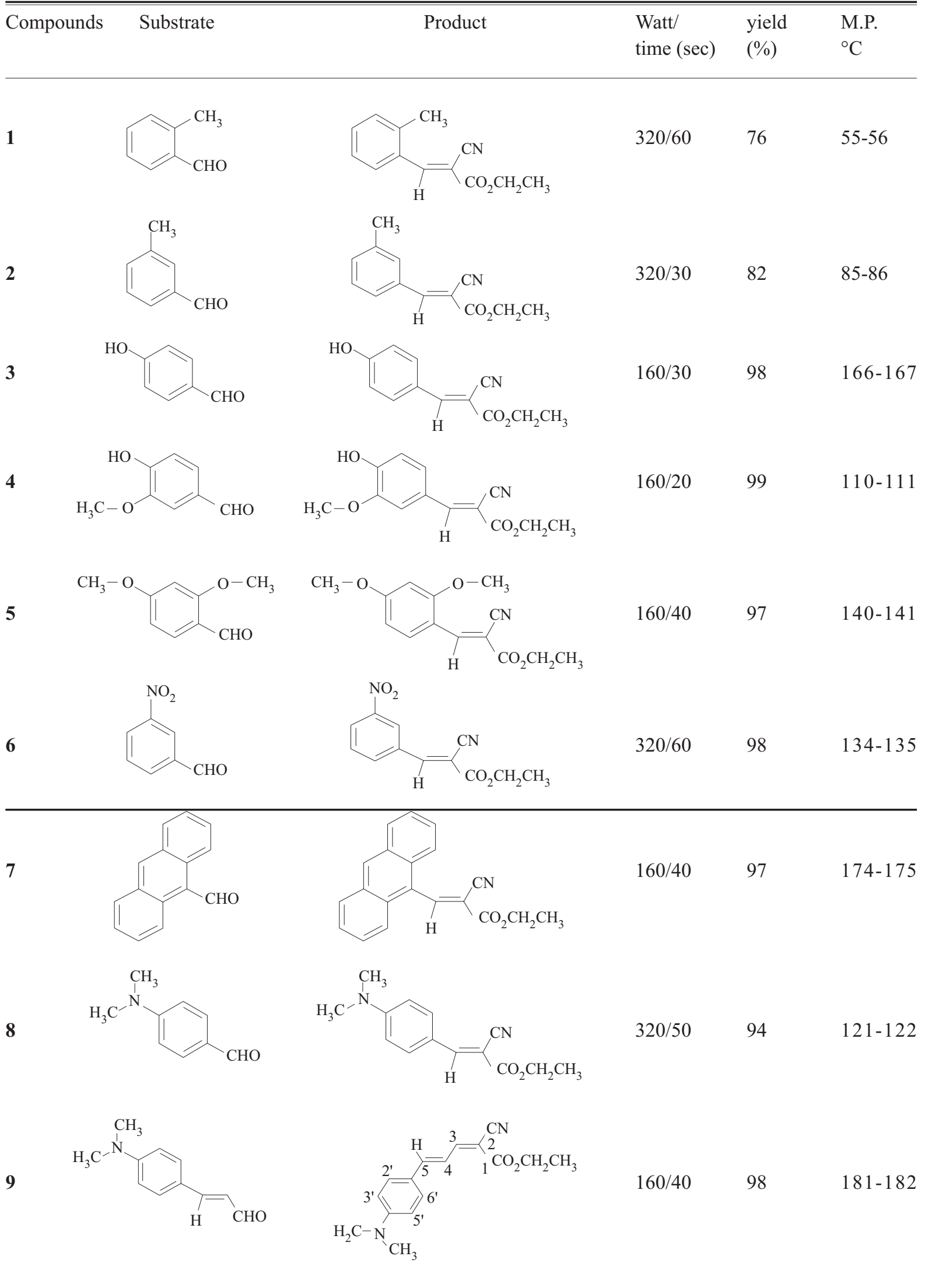


$2221.06 \mathrm{~cm}^{-1}$ was due to $\mathrm{C}=\mathrm{N}$ stretch, while bands at $1587.31 \mathrm{~cm}^{-1}, 1548.73 \mathrm{~cm}^{-1}$ were assigned to the existence of $\mathrm{C}=\mathrm{C}$, aromatic ring and a band at 1153.36 $\mathrm{cm}^{-1}$ indicated the presence of $\mathrm{C}-\mathrm{O}$ bond. The ${ }^{1} \mathrm{H}$ NMR spectrum of 9 exhibited two doublet signals resonated at $\delta 7.49$ and at $\delta 6.77$ with $J$ value $9.12 \mathrm{~Hz}$ and 9.16 $\mathrm{Hz}$ were designated to four aromatic protons of $\mathrm{H}-2$ ', H-6', H-3' and H-5'. A doublet signal at a lower field, $\delta 7.97(J=11.88 \mathrm{~Hz})$ was attributed to $\mathrm{H}-3$ of allylidene which was coupled with $\mathrm{H}-4$ in a trans-relationship. Another doublet at $\delta 7.18(J=14.9 \mathrm{~Hz})$ attributed to $\mathrm{H}-5$ of the conjugated system which was coupled with H-4 in a trans-relationship. H-4 of the conjugated system appeared as a doublet of doublet centered at d $7.11(\mathrm{~J}$ $=11.88 \mathrm{~Hz}, 2.85 \mathrm{~Hz}$ ) coupled with $\mathrm{H}-3$ and $\mathrm{H}-5$. The ${ }^{1} \mathrm{H}$ NMR spectrum also displayed a two-proton quartet at $\delta 4.31(J=7.36 \mathrm{~Hz})$ and a three proton triplet at $\delta$ $1.36(J=7.32 \mathrm{~Hz})$ which were indicative for the presence of ester group $\left(\mathrm{OCH}_{2} \mathrm{CH}_{3}\right)$ in the molecule. A six-proton singlet displayed at $\delta 3.06$ attributed to $-\mathrm{N}\left(\mathrm{CH}_{3}\right)_{2}$ group. The ${ }^{13} \mathrm{C}-\mathrm{NMR}$ spectrum of compound 9 showed the presence of thirteen signals attributed to sixteen carbons corresponding molecular formula $\mathrm{C}_{16} \mathrm{H}_{18} \mathrm{~N}_{2} \mathrm{O}_{2}$. The spectrum displayed a downfield peak at $\delta 163.19$ was assigned for $\mathrm{C}=\mathrm{O}$ of ester functionality. The peaks at $\delta 115.59,98.97,61.62,39.93,14.08$ were due to $\mathrm{CN}$ and $=\mathrm{C}<, \mathrm{OCH}_{2},-\mathrm{N}\left(\mathrm{CH}_{3}\right)_{2}$ and $\mathrm{CH}_{3}$ groups. The peaks at $\delta 156.48,152.23,150.13,130.68,122.37,117.94$, 111.6 were for the remaining carbons. The DEPT-90 spectrum of compound 9 showed peaks at $\delta 156.48$, $150.14,130.68,117.94,111.66$ attributed to seven methine carbons $(\mathrm{CH})$. Two methyl $\left(\mathrm{CH}_{3}\right)$ carbons appeared at $\delta 39.67$ and 39.65. The DEPT-135 spectra of compound 9 displayed peaks at $\delta 156.48,150.13$, $130.68,117.94,111.66$ attributed to seven methine carbons $(\mathrm{CH})$. The- $\mathrm{N}\left(\mathrm{CH}_{3}\right)_{2}$ and $\mathrm{CH}_{3}$ carbons appeared at $\delta 39.93,39.91,14.09$. The $\mathrm{OCH}_{2}$ carbon appeared at $\delta 61.63$ for $\mathrm{CH}_{2}$ as a negative value. The microanalytical data of the compound 9 is also in good agreement with the assigned structure. Similarly the peaks in ${ }^{1} \mathrm{H}$-NMR and ${ }^{13} \mathrm{C}$-NMR spectra of the rest compounds were in accordance with the assigned structures.

Amongst the compounds 1-9 screened for antimicrobial activity, compound $\mathbf{4}$ showed the highest activity against B. cereus. Most of the compounds showed good to moderate antimicrobial activities and a few of them were unable to show inhibition for some pathogens. As far as antifungal activity is concerned, all compounds showed good to excellent activity against both of the fungi. All compounds except $\mathbf{1}$ and $\mathbf{2}$ exhibited even stronger activity than Nystatin against $S$. cerevisiae.

\section{Conclusion}

In this work, the synthesis of $\alpha$-cyanoacrylates by microwave irradiation has been demonstrated using $\mathrm{NH}_{4} \mathrm{OAc}$ under and solvent free condition. The advantages of this method are high yields, relatively short reaction times, low cost, simple experimental and isolation procedures, and in agreement with the green chemistry protocols. The activity data obtained during the study will be certainly useful to go for further research for drug designing and synthesising $\alpha$-cyanoacrylates derivatives.

\section{Acknowledgment}

The authors wish to thank Dr. A. Rahman, Department of Biochemistry and Molecular Biology, University of Chittagong, Bangladesh, for his cooperation in determining the antimicrobial activity of the synthesized compounds. We also wish to thank Dr. M. Al-Amin, JSPS postdoctoral fellow, Department of Chemistry, Hokkaido University, Japan for recording the spectral data.

\section{References}

Balalaie, S., Bararjanian, M. 2006. Tetra- $n$-butylammonium hydroxide (TBAH)-catalyzed Knoevenagel Condensation: A facile synthesis of $\alpha$-cyanoacrylates, $\alpha$-cyano-acrylonitriles, and $\alpha$-cyanoacrylamides. Synthetic Communication, 36: 533-539.

Balalaie, S., Nemati, N., 2000. Ammonium acetatebasic alumina catalyzed Knoevenagel condensation under microwave irradiation under solvent-free condition. Synthetic Communication, 30: 869-875.

Basu, B., Das, P., Bhuiyan, M.M.H., Jha, S. 2003. Microwave-assisted Suzuki coupling on a KFalumina surface: synthesis of polyaryls. Tetrahedron Letters, 44: 3817-3820.

Bauer, A.W., Kirby, W.M.M., Sherris, J.C., Turck, M. 1966. Antibiotic susceptibility testing by a standardized single disc method. American Journal of Clinical Pathology, 45: 493-496.

Bhuiyan, M.M.H., Hamidunnessa, Mahmud, M.M. 2012. Multicomponent reactions: Microwave-assisted efficient synthesis of dihydropyrimidinones (thiones) and quinazolinones under green chemistry protocol as probes for antimicrobial activities. Journal of 
Scientific Research, 4: 143-153.

Bhuiyan, M.M.H., Hossain, M.I., Mahmud, M.M., AlAmin, M. 2011. Microwave-assisted efficient synthesis of chalcones as probes for antimicrobial activities. Chemistry Journal, 1: 21-28.

Bigi, F., Chesini, L., Maggi, R.R., Sartori, G. 1999. Montmorillonite KSF as an inorganic, water soluble and reusable catalyst for the Knoevenagel synthesis of coumarin-3-carboxylic acids. Journal of Organic Chemistry, 64: 1033-1035.

Campaigne, E., Schneller, S.W. 1976. Cyclization of ylidenemalonodinitriles. Synthesis, (11) 705-716.

Fatiadi, A.J. 1978a. New applications of malononitrile in organic chemistry-Part I. Synthesis, 165-204.

Fatiadi, A.J. 1978b. New applications of malononitrile in organic chemistry-Part II. Synthesis, 241-282.

Feroci, M., Orsini, M., Palombi, L., Inesi, A. 2007. Electrochemically induced Knoevenagel condensation in solvent and supporting electrolytefree conditions. Green Chemistry, 9: 323-325.

Freeman, F. 1980. Properties and reactions of ylidenemalononitriles. Chemical Review, 80: 329-350.

Grover, R.K., Moore, J.D. 1962. Toximetric studies of fungicides against the brown rot organisms Sclerotinia fructicola and S. laxa. Phytopathology, 52: 876-880.

Kandeel, Z.E., Farag, A.M., Shaaban, M.R., Elnagdi, M.H. 1996. Studies with 1,3-diketones: A convenient synthesis of some tetrahydro-4H-benzo-pyran and tetrahydroquinoline derivatives. Heteroatom Chemistry, 7: 35-38.

Kappe, C.O. 2004. Controlled microwave heating in modern organic synthesis. Angewandte Chemie International Edition, 43: 6250-6284.

Kumar, S. 2009. An efficient one-pot synthesis of 3cyanocoumarins using phase transfer catalysis. Oriental Journal of Chemistry, 25: 1145-1148.

Li, J.T., Xing, C.Y., Li, T.S. 2004. An efficient and environmentally friendly method for synthesis of arylmethylenemalononitrile catalyzed by Montmorillonite $\mathrm{K} 10-\mathrm{ZnCl}_{2}$ under ultrasound irradiation. Journal of Chemical Technology and Biotechnology, 79: 1275-1278.

Li, G., Xiao, J., Zhang, W. 2011. Knoevenagel condensation catalyzed by a tertiary-amine functionalized polyacrylonitrile fiber. Green Chemistry, 13: 1828-1836.

Lidstrom, P., Tierney, J., Wathey, B., Westman, J. 2001. Microwave assisted organic synthesis- a review. Tetrahedron, 57: 9225- 9283.

Mallouk, S., Bougrin, K., Laghzizil, A., Benhida, R. 2010. Microwave-assisted and efficient solventfree Knoevenagel condensation. A sustainable protocol using porous calcium hydroxyapatite as catalyst. Molecules, 15: 813-823.

Mukhopadhyay, C. Datta, A. 2008. A simple, efficient and green procedure for the Knoevenagel condensation of aldehydes with N-methylpiperazine at room temperature under solvent-free conditions. Synthetic Communication, 38: 2103-2112.

Parvin, M.N., Jin H., Ansari, M.B., Oh, S.M., Park, S.E. 2012. Imidazolium chloride immobilized SBA15 as a heterogenized organocatalyst for solvent free Knoevenagel condensation using microwave. Applied Catalysis A: General, 413-414: 205-212.

Saha, M., Roy, S., Chaudhuri, S.K., Bhar, S. 2008. Microwave-assisted ammonium formate-mediated Knoevenagel reaction under solvent-free conditions - a green method for C-C bond formation. Green Chemistry Letters and Reviews, 1: 113-121.

Shen, Y. 1998. New synthetic methodologies for carboncarbon double bond formation. Accounts of Chemical Research, 31: 584-592.

Tietze, L.F., Beifuss, U. 1991. The Knoevenagel reaction. In: Comprehensive Organic Synthesis, B.M. Trost, (ed.), vol. 2, pp. 341-394, Pergamon Press, Oxford, UK.

Varma, R.S. 1999. Solvent-free organic syntheses. using supported reagents and microwave irradiation. Green Chemistry, 1: 43-55.

Ye, W., Jiang, H. Yang, X.-C. 2011. Diethylamine functionalized polyethylene glycol as a novel and efficient catalyst for Knoevenagel condensation. Journal of Chemical Sciences, 123: 331-334.

Zhang, J., Jiang, T., Han, B., Zhu, A., Ma, X. 2006. Knoevenagel condensation catalyzed by $1,1,3,3-$ tetramethylguanidium lactate. Synthetic Communication, 36: 3305-3317. 